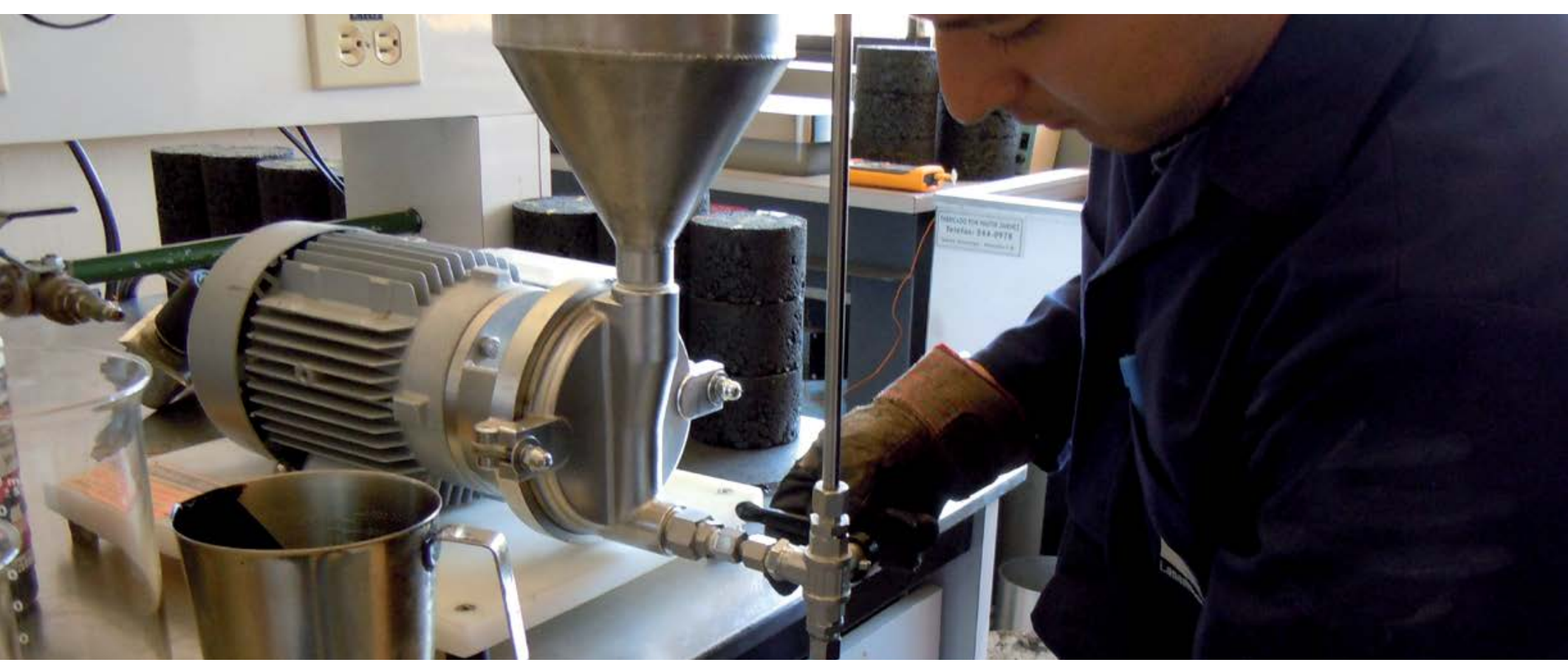

\title{
Preparación de emulsiones asfálticas en laboratorio
}

Fecha de recepción: 18 de junio del 2012

Fecha de aceptación: 30 de julio del 2012

\section{Resumen}

Costa Rica, desde hace mucho tiempo, ha hecho uso de emulsiones asfálticas para diferentes aplicaciones, sin embargo, su preparación y algunos cuidados para su almacenamiento han sido desconocidos por muchos que cotidianamente las utilizan. Por lo cual, en este artículo se detallan los procedimientos para la preparación de los diferentes tipos de emulsiones asfálticas a nivel de laboratorio, así como algunas recomendaciones de manipulación durante y posterior al proceso de fabricación, de manera que se puedan obtener los resultados esperados de acuerdo con la aplicación específica para la cual se confeccionó cada una de esas emulsiones.

\section{Palabras Claves}

Emulsiones asfálticas, fase jabonosa, emulsificante, asfalto, agua, rompimiento.
Ing. Andrea Ulloa Calderón,

Jefe del Laboratorio de Mezclas Bituminosas, LanammeUCR

Tel.: (506) 2511-2512 / E-mail: andrea.ulloacalderon@ucr.ac.cr

\section{Abstract}

Costa Rica has used asphalt emulsions for different purposes quite extensively over the years. Nevertheless, asphalt emulsion preparation and its storage care needs have been overlooked by users who employ these emulsions on a regular basis. This article intends to provide details about the procedures involved in different asphalt emulsion lab preparation. In addition, the article also gives a series of recommendations about how to properly handle these emulsions during and after the manufacturing process so that the specific desired application outcome can be achieved.

\section{Keywords}

Asphalt emulsions, soap solution, emulsifier, asphalt, water, breaking. 


\section{Introducción}

La preparación de emulsiones asfálticas se ha llevado a cabo desde inicios del siglo XX y hoy día las emulsiones son utilizadas alrededor de todo el mundo, y en Costa Rica no ha sido la excepción. Las emulsiones ofrecen gran variedad de aplicaciones en distintas técnicas para la conservación y construcción de pavimentos.

La utilización de emulsiones como alternativa de las mezclas asfálticas brinda una solución viable, por su menor costo y porque son ventajosamente más amigables con el medio ambiente, puesto que durante el proceso de preparación y en su aplicación, la cantidad de emisiones, vapores y consumo de energía es menor en comparación con las mezclas asfálticas convencionales. A pesar de ello, aproximadamente solo entre un $10 \%$ y $20 \%$ del asfalto empleado en pavimentos es usado en forma de emulsiones asfálticas.

La emulsión asfáltica se define como la combinación de tres componentes principales: asfalto, agua y una cantidad específica de un agente emulsificante, que permite la mezcla de dos sustancias que por su naturaleza química no podrían mantenerse combinadas después de ser mezcladas. Esto se logra a través de un molino coloidal o equipo especializado para separar las partículas de asfalto en tamaños suficientemente pequeños que permita combinarse con el emulsificante. Este tercer componente, el emulsificante, evita o retrasa la separación del asfalto y el agua, manteniendo estable dicha combinación en un tiempo determinado. En algunas ocasiones las emulsiones asfálticas pueden contener otros aditivos como estabilizadores, mejoradores de recubrimiento, anti-desnudantes o agentes de control de rompimiento.

Las emulsiones asfálticas se componen de un medio disperso (fase jabonosa o acuosa) en la que se mantienen

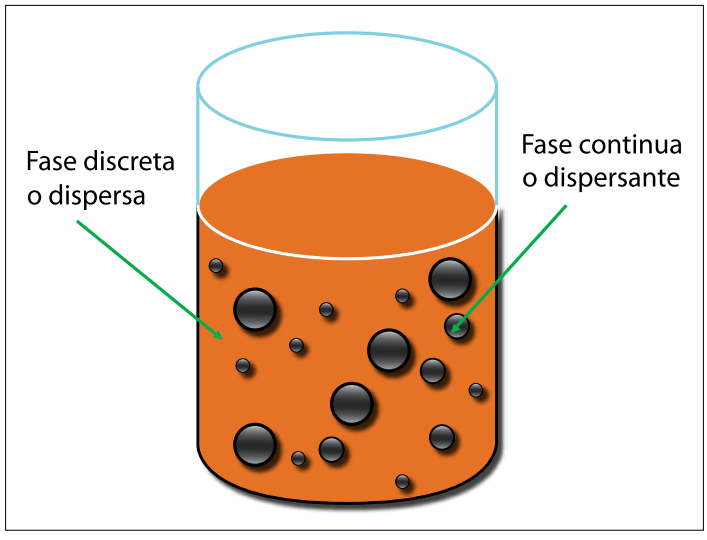

Figura 1. Diagrama de una emulsión asfáltica

separadas las partículas de asfalto o la fase dipersante, tal como se muestra en la Figura 1.

En el proceso de producción, la fase jabonosa se encuentra dispersa en el asfalto, donde las partículas de asfalto se mantienen separadas debido a que éste es reducido a pequeñas gotas que son rodeadas por una superficie de emulsificante, la cual se encuentra cargada eléctricamente y evita que éstas se atraigan nuevamente entre sí. El proceso de coalescencia o unión de las partículas de asfalto ocurre en un tiempo posterior cuando el agua contenida en la emulsión empieza a evaporarse, tal como se muestra en la Figura 2. Este fenómeno se conoce como el rompimiento o pérdida de estabilidad de la emulsión.

La producción de emulsiones asfálticas en el laboratorio, la realización de ensayos para su caracterización y el control de parámetros de acuerdo con especificaciones, se llevan a cabo de manera sencilla y económicamente accesible. Por lo que se presenta en este documento una descripción esquemática de la preparación de las emulsiones asfálticas de acuerdo con el tipo de aplicación meta; esto a partir de la experiencia técnica adquirida y las formulaciones

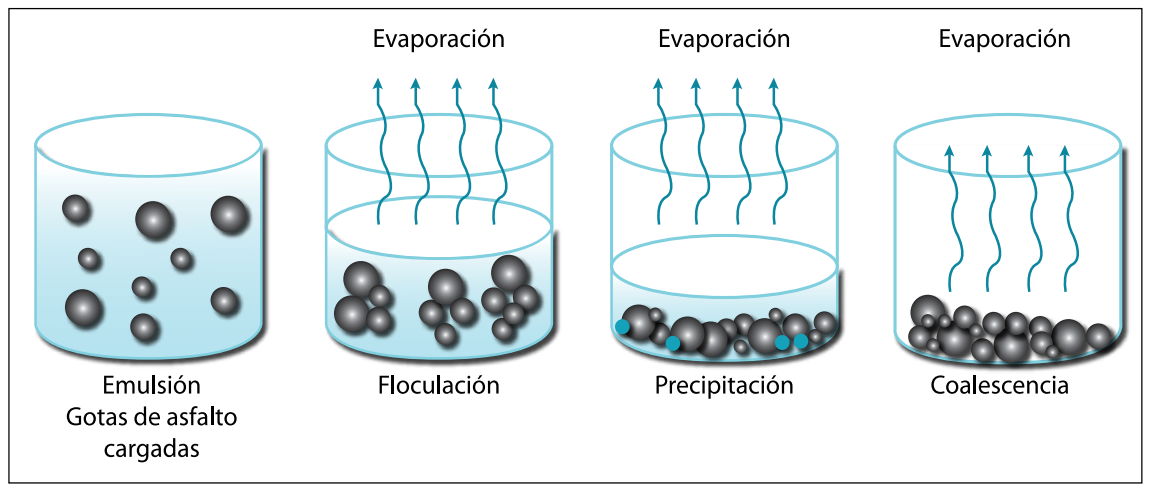

Figura 2. Etapas de la rotura en una emulsión asfáltica 
propuestas por los fabricantes de emulsificantes para cada tipo de emulsión.

\section{Tipos de emulsiones asfálticas}

Antes de establecer los tipos de emulsiones asfálticas es necesario conocer cada uno de sus componentes y entender así su comportamiento cuando se encuentran combinados.

\section{Asfalto}

La emulsión asfáltica está compuesta entre un 50\% y un 75 $\%$ de asfalto, y aún siendo el elemento predominante en la emulsión, ninguna de sus propiedades afecta la estabilidad de la misma. Sin embargo, la caracterización del asfalto es necesaria para determinar su desempeño posterior a la colocación de la emulsión. Según el clima, condiciones de tránsito esperado y el tipo de aplicación escogida, la propiedad que requiere mayor atención es la dureza del asfalto.

\section{Agua}

Ciertos minerales y algunos químicos en el agua utilizada durante la elaboración de las emulsiones, pueden afectar la estabilidad y las condiciones de almacenamiento de la emulsión asfáltica. La presencia de iones decalcioymagnesio en el agua retrasan el rompimiento de los enlaces catiónicos en emulsiones con carga positiva, contrariamente, cuando se presentan carbonatos o partículas en suspensión, se produce una pérdida de estabilidad en la emulsión debido a un prematuro rompimiento.

\section{Emulsificante}

El emulsificante está conformado una parte por cadenas de hidrocarbonos que son solubles en medios orgánicos como el asfalto y otra parte con carga eléctrica que es soluble en medios acuosos. Es por esta razón que a pesar de que el emulsificante es el componente en menor cantidad dentro de una emulsión, es quien provee las propiedades principales a la emulsión, le brinda estabilidad y determina finalmente qué tipo de emulsión asfáltica se obtendrá.

Sabiendo la importancia que posee cada uno estos componentes en una emulsión asfáltica es posible entonces clasificar las emulsiones dentro de tres categorías, según su carga eléctrica: aniónicas, catiónicas o neutras.

Las emulsiones aniónicas y catiónicas son usualmente las más utilizadas en la construcción y mantenimiento de carreteras; a diferencia de las neutras que aún son poco conocidas y que requieren mayores avances tecnológicos para ser usadas.

La carga eléctrica contenida en cada tipo de emulsificante rodea superficialmente a las partículas de asfalto, separadas por las partículas de agua, conduciendo a que se alejen entre ellas por un periodo de tiempo determinado. Ese tiempo en que las partículas de asfalto se mantienen separadas, conlleva a una segunda categorización y es de acuerdo a su reactividad (velocidad para que ocurra la coalescencia entre las partículas de asfalto), donde existe un rompimiento o pérdida de estabilidad de la emulsión. Por tanto, las emulsiones de acuerdo con esta velocidad de rotura se pueden clasificar como:

-RS (Rapid-setting): emulsiones de rompimiento rápido. -MS (Medium-setting): emulsiones de rompimiento medio.

-SS (Slow-setting): emulsiones de rompimiento lento. -QS (Quick-setting): emulsiones de rompimiento controlado.

Esta ruptura comienza en el momento que la emulsión entra en contacto con el agregado al mezclarse y cuando se coloca sobre el pavimento, debido a la separación del agua en el asfalto. La reacción química para una emulsión RS ocurre rápidamente al mezclarse con cualquier agregado, una emulsión MS es afín para mezclarse con agregado grueso pero no agregado fino, las emulsiones SS y QS por el contrario, poseen mayor afinidad para mezclarse con agregado fino, aunque la rotura en una QS se manifiesta más rápido que en una SS.

De acuerdo con las propiedades mineralógicas del agregado disponible en la mayor parte del territorio nacional, presenta mejor afinidad con las emulsiones asfálticas catiónicas para su uso en las diferentes aplicaciones de proyectos viales. Por lo tanto, en este documento los procedimientos y características mostradas en la preparación de las emulsiones asfálticas harán referencia a este tipo de emulsiones.

Es posible definir el tipo de emulsión a través de letras y números para identificarlas. Si se asigna una letra "C" delante de la nomenclatura anteriormente enunciada, se refiere a una emulsión asfáltica catiónica, por ejemplo, una CRS significa que es una emulsión catiónica de rompimiento rápido; mientras que la ausencia de alguna letra enfrente de dicha nomenclatura indica una emulsión aniónica. Si se asigna un número posterior a la nomenclatura hará referencia a la viscosidad de la emulsión; por lo que una 
CSS-2 será más viscosa que una CSS-1. Por otro lado, si se adiciona una "h" o una "s" seguido de este número indicará la dureza del asfalto utilizado, "h" para un asfalto con cierto grado de dureza y "s" para un asfalto suave.

Finalmente, si se incluye la letra "L", "S" o "P" al final de toda la nomenclatura indicará que la emulsión contiene algún aditivo o polímero, el cual suele ser látex.

\subsection{Aplicaciones}

Una última clasificación de las emulsiones asfálticas es de acuerdo con su aplicación como una técnica para la conservación de una carretera o como parte de la construcción de un pavimento. Entre los principales usos en que se pueden emplear las emulsiones se tienen las siguientes aplicaciones:

Tratamientos a la superficie

-Tratamientos superficiales (Chip seal)

- Sellos de niebla (Fogseal)

-Sellos de lechada asfáltica (Slurryseal)

-Microcapas (Microsurfacing)
-Combinación de tratamientos superficiales con sellos de lechada (Cape seal)

\section{Asfaltos reciclados}

-Reciclado en frío en sitio (Cold in-place)

-Mejoramiento de base (Full depth)

-Reciclado en caliente en sitio (Hot in-place)

-Reciclado en caliente en planta

\section{Otras aplicaciones}

-Estabilización de suelos y bases

-Bacheo

-Riego de imprimación

- Riego de liga

- Sellado de grietas

-Impermeabilizante

De forma específica, cada tipo de emulsión puede utilizarse en una o varias aplicaciones, por lo que en la Tabla 1 se exponen las diferentes aplicaciones para cada tipo de emulsión asfáltica.

Tabla 1. Técnicas de aplicaciones para cada tipo de emulsión asfáltica.

Fuente: Rererencia 1 y 3

\begin{tabular}{|c|c|c|c|c|c|c|c|}
\hline \multirow{2}{*}{$\begin{array}{c}\text { Tipo de emulsión } \\
\text { Aplicación }\end{array}$} & \multicolumn{3}{|c|}{ Aniónica } & \multicolumn{4}{|c|}{ Catiónica } \\
\hline & RS & MS & ss & CRS & CMS & css & CQS \\
\hline \multicolumn{8}{|l|}{ Riegos de emulsión } \\
\hline Tratamientos superficiales (TS) & - & & & - & & & \\
\hline Sellos de niebla & & - & $\bullet$ & & - & $\bullet$ & - \\
\hline Riego de liga & & $\bullet$ & $\bullet$ & - & $\bullet$ & $\bullet$ & \\
\hline Riego de imprimación & & $\bullet$ & $\bullet$ & & & $\bullet$ & \\
\hline \multicolumn{8}{|l|}{ Lechadas asfálticas } \\
\hline Sellos de lechada asfáltica & & $\bullet$ & - & & & $\bullet$ & $\bullet$ \\
\hline Microcapas (modificada con polímero) & & & & & & - & \\
\hline Combinación de TS + Sellos de lechada asfáltica & $\bullet$ & & & $\bullet$ & & & \\
\hline \multicolumn{8}{|l|}{ Mezcla en planta } \\
\hline Granulometría abierta / semi-densa & & - & & & $\bullet$ & & \\
\hline Granulometría densa & & $\bullet$ & $\bullet$ & & & $\bullet$ & - \\
\hline Reciclado en caliente (RAP) & & - & & & & - & - \\
\hline Bacheo & & - & & & & - & \\
\hline \multicolumn{8}{|l|}{ Mezcla en sitio } \\
\hline Reciclado en frío (RAP) & & $\bullet$ & & & $\bullet$ & $\bullet$ & $\bullet$ \\
\hline Estabilización de bases & & & $\bullet$ & & & & $\bullet$ \\
\hline Granulometría densa & & & $\bullet$ & & & $\bullet$ & $\bullet$ \\
\hline Bacheo & & - & & & - & & \\
\hline
\end{tabular}




\section{Preparación de la emulsión asfáltica}

El objetivo primordial al preparar una emulsión es producir gotas de asfalto de un tamaño lo suficientemente pequeño que puedan mantenerse en una suspensión acuosa y poder controlar el tiempo de rompimiento.

De este modo, es de suma importancia la escogencia de cada de uno de los componentes según el campo de aplicación en que se quiera utilizar la emulsión, en especial el tipo de emulsificante, que como primeramente se describió es quien define la carga iónica y el tiempo de rompimiento.

\subsection{Equipo}

El equipo utilizado para la preparación de emulsiones asfálticas tanto en el laboratorio como en planta se llama molino coloidal, el cual es un dispositivo que posee un rotor y/o rotor/estator que trabaja con un mecanismo de alta velocidad de corte, mediante el cual se logran dividir las partículas de asfalto con tamaños entre $0,25 \mathrm{~mm}$ a 0,50 $\mathrm{mm}$, en pequeñas gotas que apenas alcanzan un tamaño entre $0,001 \mathrm{~mm}$ y $0,010 \mathrm{~mm}$ máximo.

El control del tamaño y distribución de partículas en una emulsión asfáltica es relevante para determinar el desempeño de la emulsión dentro de cada aplicación, puesto que es la forma en que puede verificarse la estabilidad de la misma antes de ser aplicada en alguna técnica.

En la Figura 3 se muestra el molino coloidal y el equipo para la medición del tamaño y distribución de partículas.

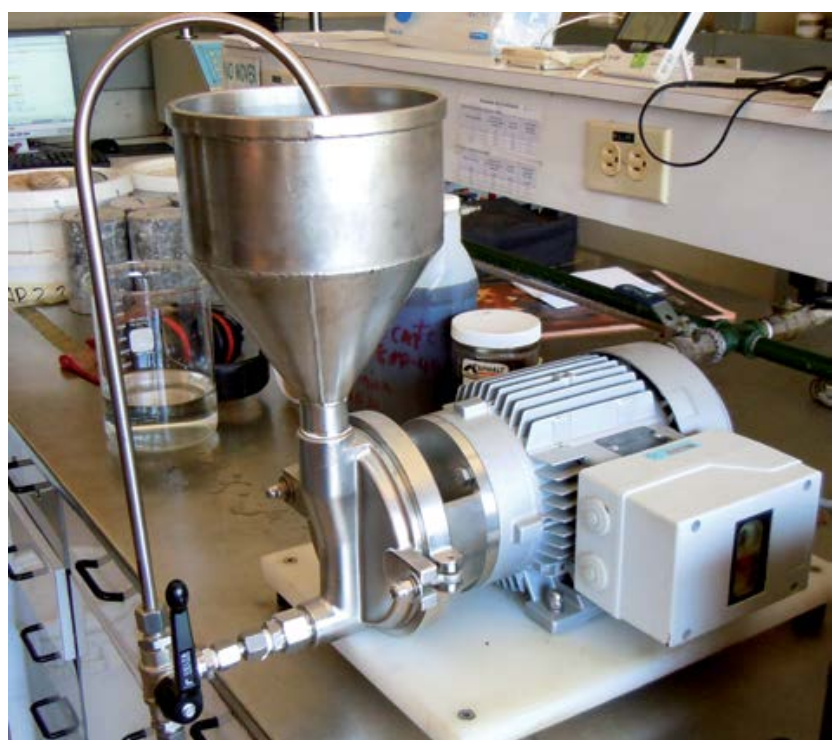

\subsection{Proceso de emulsificación}

Dado que el principal interés es la preparación de emulsiones catiónicas, se desarrollará en esta sección el procedimiento para la producción de emulsiones de este tipo. Las cuales en su mayoría son grasas aminas que se convierten en fases jabonosas al reaccionar con ácidos, en este caso el ácido usualmente utilizado para lograr dicha reacción es el ácido clorhídrico, aunque en algunas ocasiones es posible remplazar este ácido por ácido fosfórico, según el emulsificante; modificando así su tiempo de rompimiento.

El procedimiento para la preparación de una emulsión asfáltica catiónica de cualquiera de los cuatro tipos de rompimiento se resume a continuación.

\section{-Formulación}

Es indispensable inicialmente contar con la formulación que suministra la ficha técnica de cada emulsificante, dado que en ella se especifica las cantidades porcentuales óptimas para la dosificación de agua, emulsificante, asfalto y polímero en caso que se requiera modificar la emulsión. Igualmente, se indicará las temperaturas máximas de trabajo, el proceso de dosificación y si se requiere o no acondicionamiento antes de ser envasada y almacenada.

La preparación se realiza para un lote teórico de $1000 \mathrm{~mL}$ de emulsión asfáltica, pues por lo general los dispositivos usados a nivel de laboratorio en la fabricación de emulsiones poseen esa capacidad máxima por vez por lote.

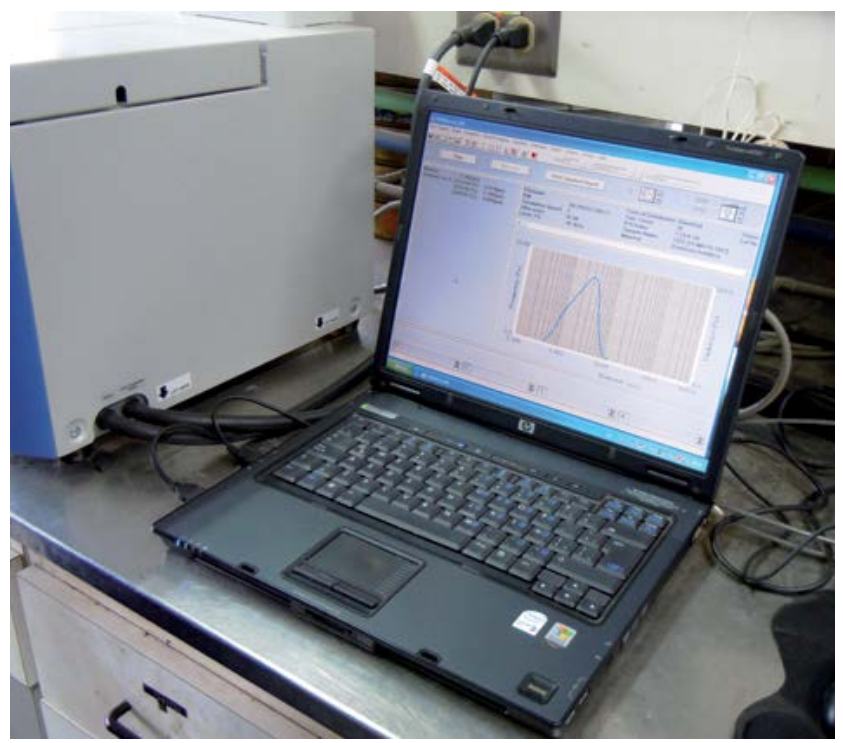

Figura 3. Equipo utilizado para la fabricación de emulsiones y la verificación del tamaño de partículas 
Los rangos porcentuales típicos de cada uno de los componentes para la formulación se muestran en la Tabla 3.

Tabla 3. Porcentajes de dosificación típicos para la preparación de una emuslión asfáltica

\begin{tabular}{|c|c|c|c|c|}
\cline { 2 - 5 } \multicolumn{1}{c|}{} & \multicolumn{4}{c|}{ Rangos de dosificación (\%) } \\
\hline Ingrediente & CRS & CMS & CSS & CQS \\
\hline Asfalto & 65 & 60 & $60-65$ & $60-65$ \\
\hline Emulsificante & $0.1-0.25$ & $0.4-0.6$ & $1.5-2.5$ & $0.8-1.8$ \\
\hline $\begin{array}{c}\text { Polímero (Aditivo) } \\
\text { (sobre el peso del asfalto) }\end{array}$ & - & - & 3 & 3 \\
\hline Agua & \multicolumn{4}{c|}{ Hasta completar el $100 \%=$} \\
\hline Ácido (HCl ó $\mathrm{H}_{3} \mathrm{PO}_{4}$ ) & $2-4$ & $2-3$ & $1.5-2.5$ & $1.5-2.5$ \\
\hline $\begin{array}{c}\text { Fase jabonosa (Agua, } \\
\text { Emulsificante y ácido) }\end{array}$ & \multicolumn{5}{c}{ Asfalto + \%Emulsificante + \%Polímero) } \\
\hline
\end{tabular}

-Preparación de la fase jabonosa

Dado que el molino coloidal debe pre-acondicionarse, es necesario preparar una cantidad mayor de fase jabonosa, por lo general y de acuerdo con la experiencia, se producen $1000 \mathrm{ml}$ de fase jabonosa, de los cuales aproximadamente un $60 \%$ es utilizado para la limpieza y acondicionamiento del molino coloidal.

La dosificación se lleva a cabo mediante pesaje de cada adición en una relación masa/masa $(\mathrm{m} / \mathrm{m})$, por lo que es necesario determinar inicialmente los porcentajes $(\mathrm{m} / \mathrm{m})$ y con ello los pesos de dosificación a partir de la formulación propuesta para el emulsificante escogido. De este modo el procedimiento recomendado a seguir para la preparación de la fase se muestra en la figura 4.

\section{-Asfalto}

El asfalto debe calentarse a la temperatura previamente establecida en la caracterización de sus propiedades, para lograr bajar su viscosidad y que este sea trabajable. Dicha temperatura se encuentra en un rango entre $125^{\circ} \mathrm{C}$ y $140{ }^{\circ} \mathrm{C}$, de modo que al mezclarse con la fase jabonosa, no supere los $85^{\circ} \mathrm{C}$, puesto que de superar los $100{ }^{\circ} \mathrm{C}$ el agua contenida en la fase se evapora y acelera el proceso de rompimiento de la emulsión. La escogencia de un asfalto muy duro o modificado con algún aditivo, requiere un mayor control durante el proceso de producción, dado que se incrementará la temperatura, aumentando la posibilidad de que el agua alcance el punto de ebullición.
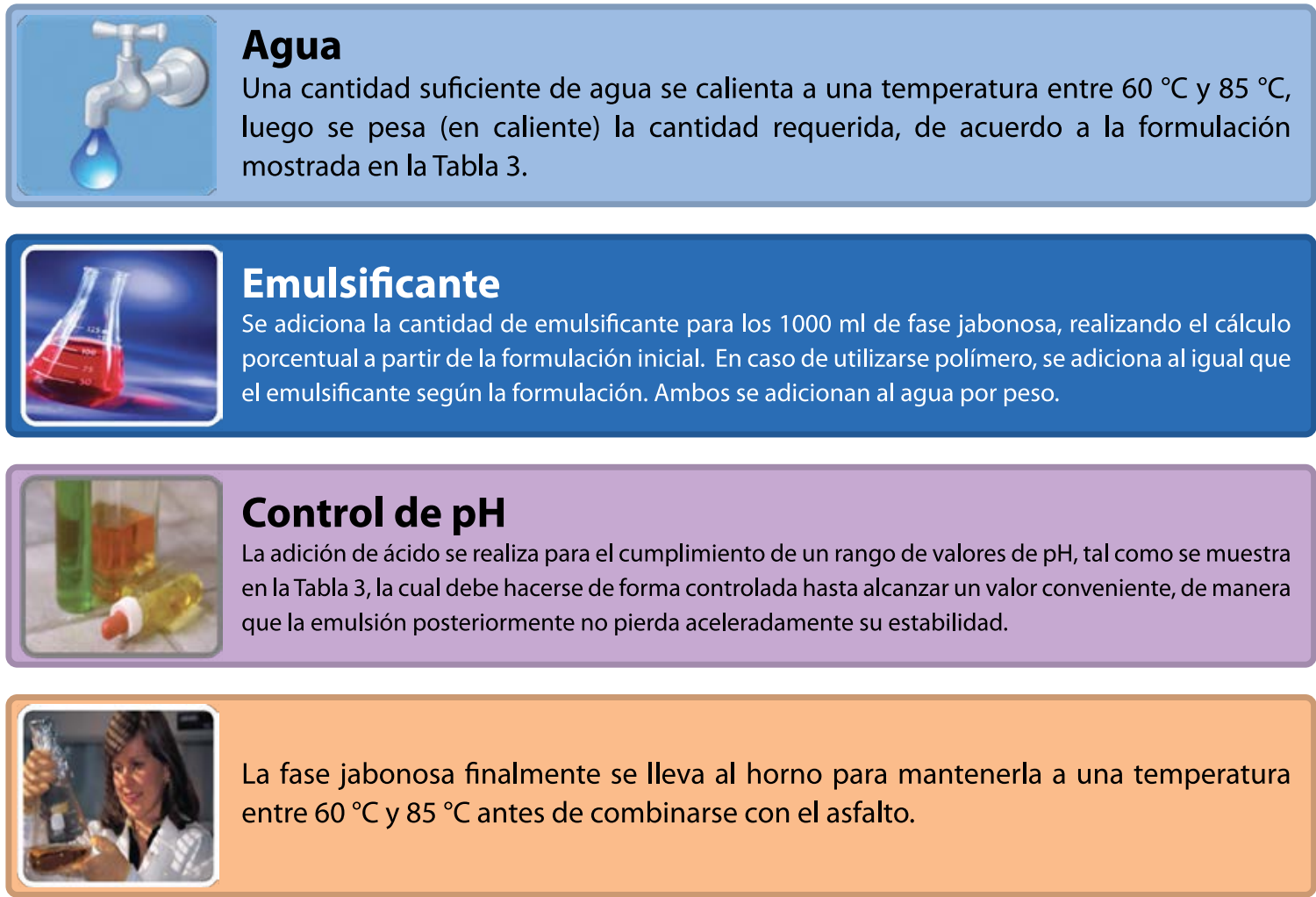

Figura 4. Etapas para la preparación de la fase jabonosa 
-Mezclado y pre-acondicionamiento del equipo

Teniendo en consideración lo anterior, se necesita primeramente tomar en cuenta que la temperatura de trabajo o de mezclado en el molino coloidal debe estar entre $85^{\circ} \mathrm{C}$ y $95^{\circ} \mathrm{C}$ para evitar una evaporación importante del agua.

Para esto se adicionan de $500 \mathrm{ml}$ a $600 \mathrm{ml}$ de la fase jabonosa al molino, misma que se hallaba en el horno a una temperatura de $60{ }^{\circ} \mathrm{C}$ aproximadamente. Esto se usa para limpiar y calentar el molino coloidal, antes de realizar el mezclado.

Posterior a esto, se cierra la válvula de salida y se adiciona la fase jabonosa (previamente pesada) de acuerdo con la formulación para los 1000 ml de emulsión.

Se activa el motor y se hace circular por unos segundos la fase jabonosa. Se adiciona el asfalto de manera que el grosor del flujo vertido no supere el diámetro de una pajilla (aproximadamente $6 \mathrm{~mm}$ ). Esta acción no debe superar los 60 segundos, pues podría reducir en exceso el tamaño de las partículas del asfalto, al igual que sobrecalentar tanto el equipo como la combinación misma.

Se busca que la temperatura de salida no supere los $90{ }^{\circ} \mathrm{C}$ al concluir el mezclado.

\section{-Almacenamiento}

La emulsión asfáltica debe ser almacenada en envases esbeltos, cilindricos y con un cuello estrecho para su cierre, de manera que no haya pérdida de volátiles o que el agua se evapore rápidamente, ocasionando la pérdida de la estabilidad antes de lo previsto.

\section{-Acondicionamiento o curado de la emulsión}

Cuando el productor del emulsificante así lo establezca por las características químicas del mismo, se deberá acondicionar la emulsión por 24 horas en un horno a 60 ${ }^{\circ} \mathrm{C}$ aproximadamente, antes de ser almacenada de la forma descrita anteriormente. Sin embargo, esto es aplicable únicamente para emulsiones de rompimiento lento (CSS), debido a que requieren un periodo más prolongando para que el proceso químico concluya, antes de poderse utilizar en alguna de las aplicaciones.
En la Figura 5 se ejemplifica el proceso de preparación de una emulsión asfáltica catiónica de rompimiento lento, CSS-1h.

\section{Recomendaciones}

Para seleccionar el tipo de emulsión asfáltica adecuado es necesario estudiar las propiedades químicas del agregado (principalmente la carga iónica y/o sus propiedades mineralógicas) y el sistema constructivo involucrado.

Para obtener un buen desempeño de las emulsiones asfálticas en las diferentes aplicaciones, es importante conocer previamente las características de los materiales y qué factores pueden afectar la preparación la emulsión, entre los cuales se tienen:

- Las propiedades químicas y dureza del asfalto

-El tamaño de partículas del asfalto en la emulsión

- La cantidad de emulsificante

- Las condiciones de manipulación y temperatura en el proceso de fabricación

- La carga iónica de las partículas esperada en la emulsión

-El orden en que se adicionan los componentes

- Las características y la cantidad adicionada de modificadores o polímeros

- La dureza del agua

El control de las dosificaciones de acuerdo a la formulación, así como la verificación de las temperaturas durante el proceso de producción permiten que la emulsión asfáltica mantenga sus propiedades químicas durante el tiempo previsto.

\section{Referencias}

1. AkzoNobel. Emulsiones bituminosas. Documento técnico.

2. Asphalt Emulsion Manufacturers Association. The Basics of Asphalt Emulsions. Documento técnico.

3. Asphalt Institute. A Basic Asphalt Emulsion Manual. Manual Series No. 19 (MS-19), 1979.

4. Rodríguez Talavera, Rogelio; Castaño Meneses, Víctor Manuel y Martínez Madrid, Miguel. Emulsiones Asfálticas. Secretaría de Comunicaciones y Trasportes, Instituto Mexicano del Transporte. Documento Técnico No. 23; Sanfandila, Qro, 2001. 


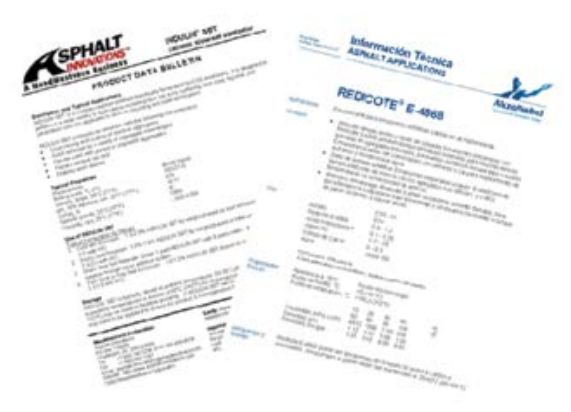

(a) Formuliación a partir de las fichas técnicas del emulsificante

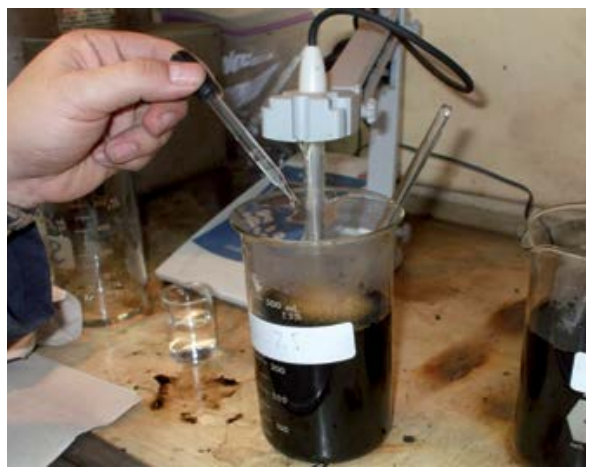

(d) Adición de $\mathrm{HCl}$, controlando el pH

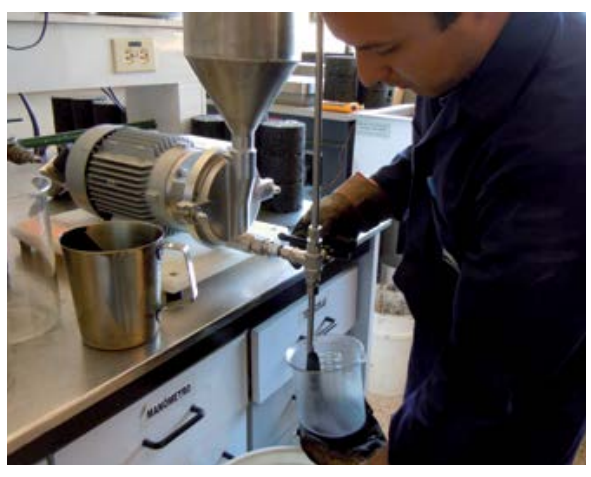

(g) Salida de emulsión a $95^{\circ} \mathrm{C}$

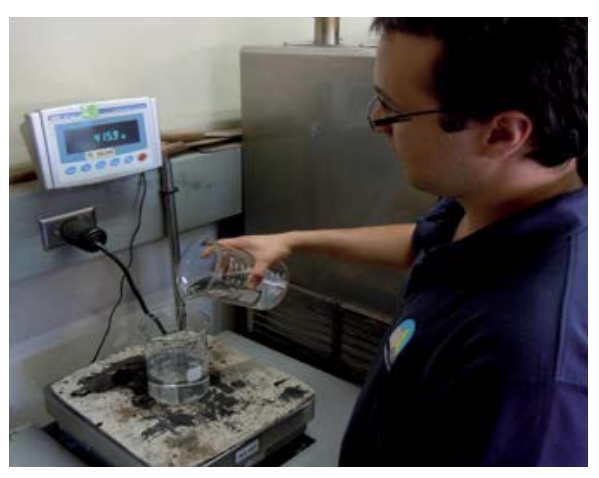

(b) Medición por peso del agua

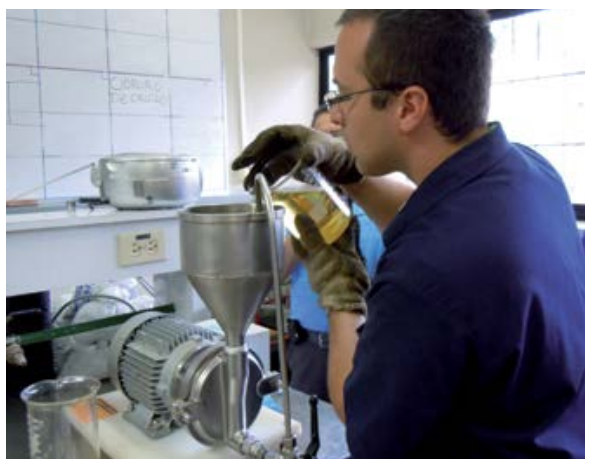

(e) Limpieza y acondicionamiento del molino coloidal

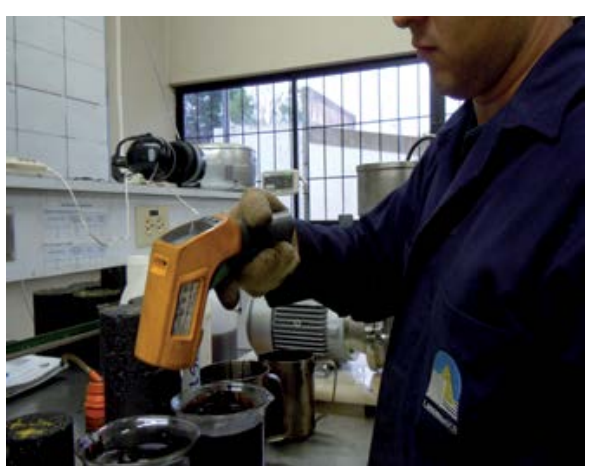

(h) Acondicionamiento y almacenamiento

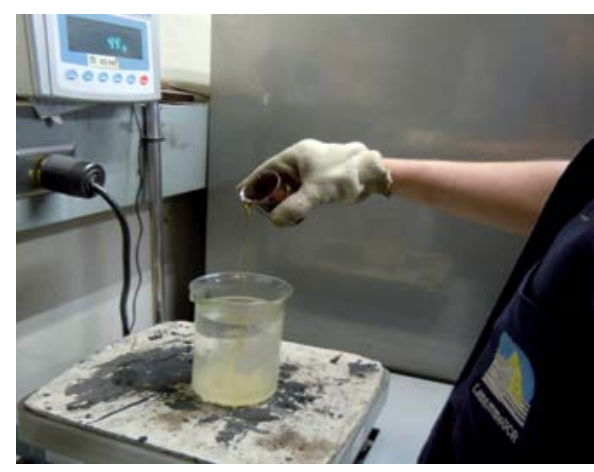

(c) Adición del emulsificante para una CSS-1h

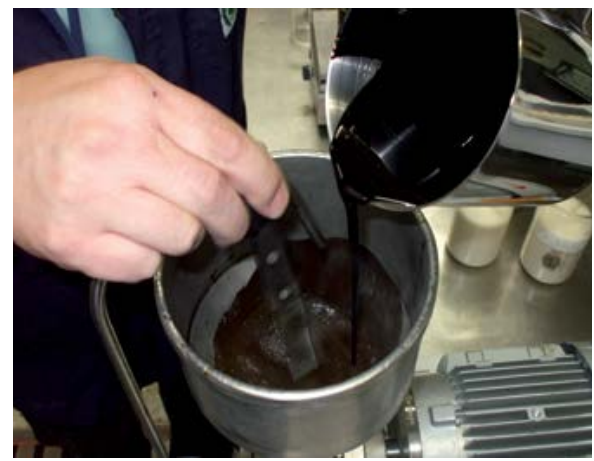

(f) Mezclado de fase jabonosa y asfalto en el molino coloidal

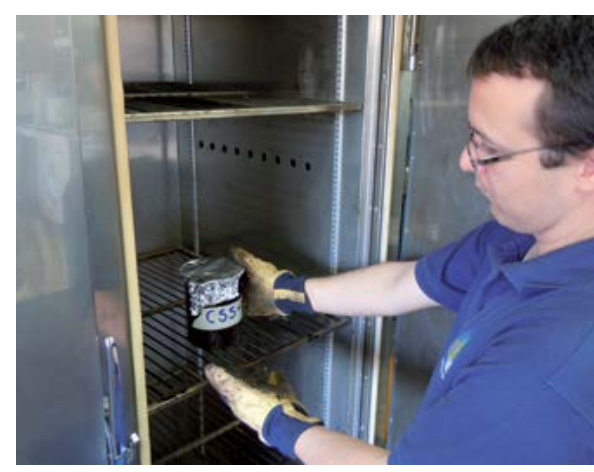

Figura 5. Etapas de la preparación de una emulsión asfáltica 